Falsch negativer Sentinel

\title{
Melanom: Was passiert, wenn der Wächter schläft?
}

In ihrer retrospektiven Studie gingen Edward Jones, Denver, CO/USA, und sein Team unter anderem der Frage nach, wie die Erkrankung nach Melanomrezidiven trotz negativen Sentinels voranschreitet. Dafür analysierten sie die Krankenblätter

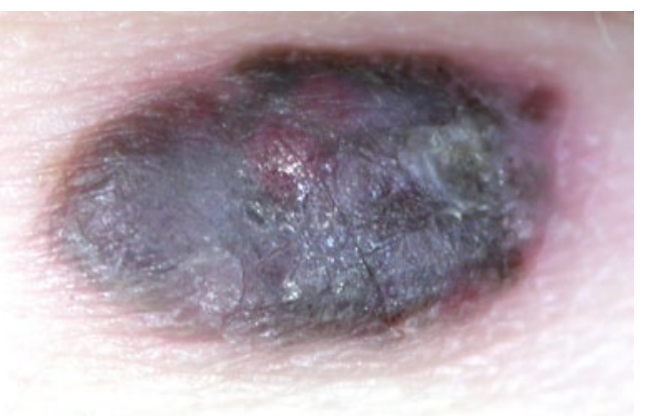

Melanomrezidive können auch noch nach acht Jahren auftreten. von 515 Melanompatienten, die zwischen 1996 und 2008 eine lokale Tumorexzision mit begleitender Biopsie von Wächterlymphknoten erhalten hatten. Melanomrezidive erlitten 83 Patienten (16\%). $21 \mathrm{~Pa}$ tienten zeigten dabei Metastasen im Abstromgebiet der Sentinelknoten. Bezogen auf die Gesamtzahl von 515 Patienten betrug die Rate falsch negativer Befunde damit rund $4 \%$. Von Rückfällen betroffen waren besonders Patienten mit tieferen Primärläsionen: Im Durchschnitt erreichte die Breslow-Dicke der ursprünglichen Läsion 2,8 mm bei Patienten mit und 1,8 mm bei Patienten ohne Rezidiv. Auch wiesen die Patienten, die später ein Melanomrezidiv entwickelten, häufiger ulzerierte Läsionen auf (32,5 vs. 13,5\%). Anfällig dafür, Absiedlungen zu bilden, waren Melanome im Kopf- und Halsbereich - 42,2\% der Rezidiv-, aber nur 17,2\% der von Rezidiven verschonten Patienten hatten zuvor Melanome in diesem Bereich aufgewiesen. Höheres Lebensalter bei Diagnose war allerdings mit schwachem Zusammenhang - ebenfalls häufiger mit Rückfällen assoziiert. Das Auftreten von Melanommetastasen verschlechterte die Prognose deutlich. Lag die 5-Jahres-Überlebensrate in der gesamten Gruppe mit negativem Sentinelbefund bei $91 \%$, sank sie nach Auftreten von Rezidiven auf $64 \%$. Die Lokalisation spielte dabei keine wesentliche Rolle. Erhellend war auch ein Blick auf den Zeitpunkt des erneuten Melanomwachstums. Im Median trat das Rezidiv nach knapp zwei Jahren auf, es konnte sich aber auch mehr als acht Jahre nach der ersten Diagnose noch entwickeln. Hätte man die Patienten nur fünf Jahre lang nachuntersucht, wären fast $11 \%$ der Rückfälle übersehen worden.

Robert Bublak

Jones EL et al. Long-term Follow-up and Survival of Patients Following a Recurrence of Melanoma After a Negative Sentinel Lymph Node Biopsy Result. JAMA Surg. 2013 Jan 16:1-6. [Epub ahead of print]

\section{Unveränderte Rate an Zweittumoren}

In Studien wurde ein Zusammenhang zwischen einem Androgenentzug bei Prostatakarzinompatienten und einem erhöhten Risiko für Osteoporose, Knochenbrüche und kardiovaskuläre Erkrankungen beschrieben. Bekannt ist ein erhöhtes Risiko für Hyperinsulinämie, Diabetes mellitus und Adipositas. 2010 war in einer Studie ein Zusammenhang zwischen der Hormontherapie und der Entstehung eines kolorektalen Karzinoms festgestellt worden. Das Risiko stieg mit der Dauer der Hormontherapie [J Natl Cancer Inst. 2010;102(23):1760-70]. Um diese Assoziation zu untersuchen, analysierten Wissenschaftler nun SEER (Surveillance, Epidemiology and End Results)-Daten von Männern, die zwischen 1998-2007 ein lokalisiertes Prostatakarzinom diagnostiziert bekamen. Das Follow-up endete 2009. Daten von insgesamt 24.034 zum Diagnosezeitpunkt mindestens 18-jährigen Männern wurden retrospektiv untersucht. 1.359 Männer (5,7\%) mit lokal begrenztem Prostatakarzinom entwickelten Zweittumoren (darunter 187 Kolorektal-, 178 Lungen-, 132 Blasen- und 76 Nierentumoren). Sie waren älter und hatten ein größeres Risiko für Typ-2-Diabetes oder Bluthochdruck als jene Patienten, die nur ein Prostatakarzinom hatten. Nach u.a. Berücksichtigung von Alter, ethnischer Zugehörigkeit, Jahr der Prostata-Ca.-Diagnose, Tumorstadi- um, Gleason-Score und Radiotherapie ergab ein Vergleich der Patientengruppen mit und ohne Androgenentzug keinen statistisch signifikanten Unterschied. Auch Diabetes und Adipositas hätten den Zusammenhang zwischen Zweittumor und Hormontherapie nicht beeinflusst. Peter Leiner

Wallner LP et al. Cancer and Risk of Second Primary Malignancies Androgen Deprivation Therapy for Treatment of Localized Prostate Cancer and Risk of Second Primary Malignancies. Cancer Epidemiol Biomarkers Prev. 2013;22(2):313-6.

\section{kurz notiert}

Ausschreibung Nachsorgepreis der Deutschen Kinderkrebsnachsorge

Die Deutsche Kinderkrebsnachsorge - Stiftung für das chronisch kranke Kind vergibt zum 12. Mal den mit 10.000 Euro dotierten Nachsorgepreis für zukunftsweisende Arbeiten, Projekte und Initiativen der stationären und ambulanten familienorientierten Nachsorge und Betreuung. Mit dieser Auszeichnung sollen sowohl wissenschaftliche als auch praktische zukunftsweisende Arbeiten, Projekte und Initiativen der stationären und ambulanten familienorientierten Nachsorge und Betreuung gefördert werden.
Der Preis kann an Einzelpersonen, Arbeitsgruppen, Initiativen sowie Verbände aus dem deutschen Sprachraum vergeben werden. Die Verleihung des Nachsorgepreises erfolgt Ende des Jahres 2013.

Die Bewerbungsfrist endet am 31. Juli 2013 Weitere Informationen im Internet www.kinderkrebsnachsorge.de per Mail: info@kinderkrebsnachsorge.de oder per Telefon unter 07705/920-182 